\title{
¿Es posible graficar soluciones de ecuaciones diferenciales sin necesidad de derivar o integrar? ${ }^{1}$
}

\author{
Is it possible to graph solutions of differential \\ equations without referral or integrate?
}

\author{
É possível soluções gráficas de equações \\ diferenciais sem referência ou integrar?
}

Recibido: mayo de 2013

Aceptado: agosto de 2013

\section{Resumen}

Para solucionar ecuaciones diferenciales es fundamental el uso de las integrales o de las derivadas, pero si queremos comprobar una de sus soluciones, en este documento se encontrará una manera alternativa de hacerlo teniendo en cuenta algunos aspectos como: qué es la pendiente de una recta y cómo se grafica, conocer algunas funciones y sus gráficas, tener una noción de derivada como la pendiente de una recta tangente a una curva, sabiendo esto es posible dar una solución aproximada a una ecuación diferencial haciendo un bosquejo de la función que al derivarse resultará la ecuación diferencial propuesta.

Palabras clave: Matemáticas superiores; Cálculo; Campos direccionales; Ecuaciones diferenciales; Otras nociones de Educación Matemática; Sistemas de representación; Gráficas, funciones.

\section{Abstract}

To solve differential equations is essential to use integrals or derivatives, but if we want to check one of their solutions, this paper will be an alternative way to do considering some aspects such as: what is the slope of a line and how is plotted, meet some functions and their graphs, have a notion of derivative as the slope of a line tangent to a curve, knowing this can give an approximate solution to a differential equation by an outline of the role that the result will be the differential equation given.

Keyword: higher mathematics, Calculus, Campos directional, differential equations; Other notions of Mathematics Education; systems of representation; Charts, functions.

\section{Resumo}

Para resolver equações diferenciais é essencial usar integrais ou seus derivados, mas se quiser conferir uma de suas soluções, este trabalho será uma forma alternativa de se considerar alguns aspectos, tais como: qual é a inclinação de uma linha e como é plotado, conhecer algumas funções e seus 
gráficos, ter uma noção de derivada como a inclinação de uma reta tangente a uma curva, sabendo que isso pode dar uma solução aproximada para a equação diferencial por um esboço do papel que o resultado será o equação diferencial dada.

Palabras clave: Matemática Superior, Cálculo, Campos direcional, equações diferenciais, outras noções de Educação Matemática, sistemas de representação, gráficos, funções.

\section{Planteamiento del problema}

En el primer semestre del 2013 en la cátedra Matemática del movimiento III de octavo semestre del proyecto curricular de Licenciatura en Educación Básica con Énfasis en Matemáticas en la Universidad Distrital Francisco José de Caldas (Bogotá, Colombia), se proponen actividades enmarcadas en la resolución de problemas, donde implícitamente se hizo una aproximación a la solución de ecuaciones diferenciales, inicialmente se propone la ecuación $y^{\prime}=y$ sin hablar de ecuaciones diferenciales, simplemente la idea es encontrar la solución de esta ecuación, teniendo en cuenta esto, se propone encontrar una función, tal que su derivada sea igual a la función, donde en primera instancia no es absolutamente necesario hacer un proceso de integración para dar solución, a continuación se encontrará un marco de referencia al respecto donde se sustentan otros aspectos diferente a la integral para dar solución a ecuaciones de este estilo, posteriormente se darán algunos procesos para encontrar estas soluciones sin hacer uso de la integral.

\section{Marco de referencia conceptual}

Para tratar la solución de ecuaciones diferenciales sin hacer uso de las integrales o las derivadas, aunque en este caso es necesario tratar aspectos propios de la teoría cualitativa de las ecuaciones diferenciales ya que si se tocan los aspectos analíticos se recurriría a la integral.

Para hacer un tratamiento de la teoría cualitativa de las ecuaciones diferenciales se tendrá en cuenta algunos planteamientos realizados por Cánovas (2004), para esto, inicialmente se tiene en cuenta que las

ecuaciones diferenciales ordinarias pueden ser abordadas con mayor facilidad haciendo uso de campos direccionales, estos son planos donde aparecen pendientes que indican la dirección de la tangente a una función en cada punto. El conjunto de los segmentos de esas pequeñas rectas es la representación geométrica del campo de pendientes o campo direccional del cual se puede esbozar la gráfica.

Observemos las ecuaciones del tipo $\mathrm{dx}=f(\mathrm{t})$. Para este tipo de ecuaciones se cumple en $d t$ que la pendiente en cualquier punto es la misma que en cualquier otro punto con la misma coordenada $t$. Geométricamente esto implica que todas las marcas de pendientes sobre cada $t$ son paralelas. Encontrar soluciones para este tipo de ecuaciones diferenciales es como calcular una antiderivada aunque de manera geométrica. Por ejemplo estudiemos el campo direccional de la ecuación $y^{\prime}=2 t$ la solución exacta a partir de una integral se tiene que es $\mathrm{y}=\mathrm{t}^{2} / 2+\mathrm{c}$, y las curvas solución que no son diferentes sólo es una traslación vertical; el campo direccional de esta ecuación diferencial corresponde al siguiente:

\begin{tabular}{|c|c|c|c|c|c|c|c|c|c|c|c|c|c|}
\hline 1 & 1 & 1 & 1 & 1 & 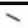 & -1 & - & - & 7 & 1 & 1 & / & 1 \\
\hline 1 & 1 & 1 & 1 & 1 & - & - & - & - & , & , & I & I & I \\
\hline 1 & 1 & 1 & 1 & 1 & - & - & - & - & , & , & I & 1 & I \\
\hline 1 & 1 & 1 & 1 & 1 & - & - & - & - & , & , & I & 1 & I \\
\hline 1 & 1 & 1 & 1 & 1 & - & - & - & - & , & 1 & 1 & 1 & I \\
\hline 1 & 1 & 1 & 1 & $\checkmark$ & $\gamma$ & $\overline{05}$ & - & - & , & , & I & I & I \\
\hline 1 & 1 & 1 & 1 & 1 & - & - & - & - & - & ' & ' & 1 & 1 \\
\hline 1 & 1 & 1 & 1 & 1 & - & - & - & - & ' & 1 & ' & 1 & I \\
\hline 1 & 1 & 1 & 1 & 1 & - & - & - & - & ' & ' & 1 & 1 & 1 \\
\hline 1 & 1 & 1 & 1 & 1 & - & - & - & - & , & ' & ' & I & I \\
\hline 1 & 1 & 1 & 1 & 1 & - & 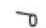 & - & - & , & 1 & 1 & 1 & 1 \\
\hline$T_{-1}^{1}$ & $T$ & $T$ & & & $\checkmark$ & $=$ & $\sigma$ & - & ' & 5 & 1 & $T$ & ${ }_{1}$ \\
\hline 1 & 1 & 1 & 1 & 1 & - & - & - & - & , & , & I & 1 & 1 \\
\hline 1 & 1 & 1 & 1 & $\checkmark$ & - & - & - & - & , & , & I & 1 & 1 \\
\hline 1 & 1 & 1 & 1 & 1 & - & - & - & - & , & , & I & 1 & 1 \\
\hline 1 & 1 & 1 & 1 & 1 & - & - & - & - & , & 1 & 1 & 1 & 1 \\
\hline 1 & 1 & 1 & 1 & 1 & - & -0.5 & - & - & , & , & I & 1 & 1 \\
\hline 1 & 1 & 1 & 1 & 1 & - & - & - & - & , & , & 1 & 1 & 1 \\
\hline 1 & 1 & 1 & 1 & 1 & - & - & - & - & , & 1 & 1 & 1 & 1 \\
\hline 1 & 1 & 1 & 1 & 1 & - & - & - & - & , & 1 & I & 1 & 1 \\
\hline 1 & 1 & 1 & 1 & 1 & - & - & - & - & , & 1 & 1 & 1 & 1 \\
\hline 1 & 1 & 1 & 1 & 1 & - & $\Rightarrow 1$ & - & - & , & ' & 1 & 1 & 1 \\
\hline & 1 & 1 & 1 & 1 & 2 & - & - & - & 1 & 1 & 1 & & \\
\hline
\end{tabular}

Fuente: Elaboración propia 
Encontrando de esta manera las posibles soluciones de la ecuación, dando un bosquejo de parábolas iguales pero trasladadas de manera vertical. Además es importante tener en cuenta que se cortan y esto se debe al teorema de existencia y unicidad de soluciones, y en la gráfica es evidente que también se da por ser una traslación de una función.

Partiendo de esto y tal como lo indica Amaya (2006) establece que "no es posible estudiar los fenómenos relativos al conocimiento sin recurrir a la noción de representación, y esto, porque no hay conocimiento que un sujeto pueda movilizar sin una actividad de representación", ahora para (Duval 1999) citado por Amaya (2006) el hecho de poder utilizar diferentes planos de representación y procedimientos para favorecen el aprendizaje de las matemáticas, esto incrementa notoriamente que el estudiante logre comprender mejor la temática a trabajar; dado que permite al docente ampliar las alternativas didácticas y procedimentales frente a la presentación de cada tema.

En el caso del cálculo por ejemplo, los conceptos de función, límite, continuidad, derivada, integral, y en este caso ecuaciones diferenciales, es posible estudiarlos acudiendo al plano de representación gráfico o tabular.

\section{Metodología}

Para llevar a cabo la solución de ecuaciones diferenciales sin hacer uso de derivar o integrar se realizará un paso a paso de los aspectos que intervienen además de una justificación.

Tengamos en cuenta que tal como se indicó en el marco referencial, el uso de tablas puede ayudar a comprender de buena manera lo que se está realizando en cálculo, en el siguiente ejemplo se mostrará el procedimiento que se realiza para la solución de una ecuación diferencial partiendo de su campo direccional.

Realicemos por ejemplo la ecuación $\mathrm{y}^{\prime}=\mathrm{y}$ donde se debe encontrar una función, tal que su derivada sea igual a la función, en este caso la variable de pendiente es y' tendremos lo siguiente:

Figura 1

\begin{tabular}{|c|c|}
\hline$y$ & $y$ \\
\hline 0 & 0 \\
\hline 1 & 1 \\
\hline
\end{tabular}

Fuente: Elaboración propia

Nótese que sí: y es igual a cero $y^{\prime}$ también lo es, si $y=1$ $y^{\prime}=1$ de esta manera se puede seguir tabulando y se encontrará que si $y=n$ entonces $y^{\prime}=n$, claro está que se da exclusivamente para la solución de esta ecuación.

Pero es necesario saber que y' es la derivada de la función, esto indica que la pendiente de la recta tangente a la función $y=f(x)$ por un punto determinado es cada uno de los valores que se da en la tabla, pero se sabe también que $y=0 . y=1 \ldots y=n$ son rectas horizontales, por lo tanto al tener en cuenta los dos valores que se dan en la tabla a la hora de tabular se interpreta que: por la recta $y=n$ se pondrán pequeños segmentos de recta con pendiente $n$, es decir por $y=0$ se tomarán pedacitos de pendiente 0 , por $y=1$ se obtendrán pedacitos de pendiente 1 y así sucesivamente de la siguiente manera:

Figura 3

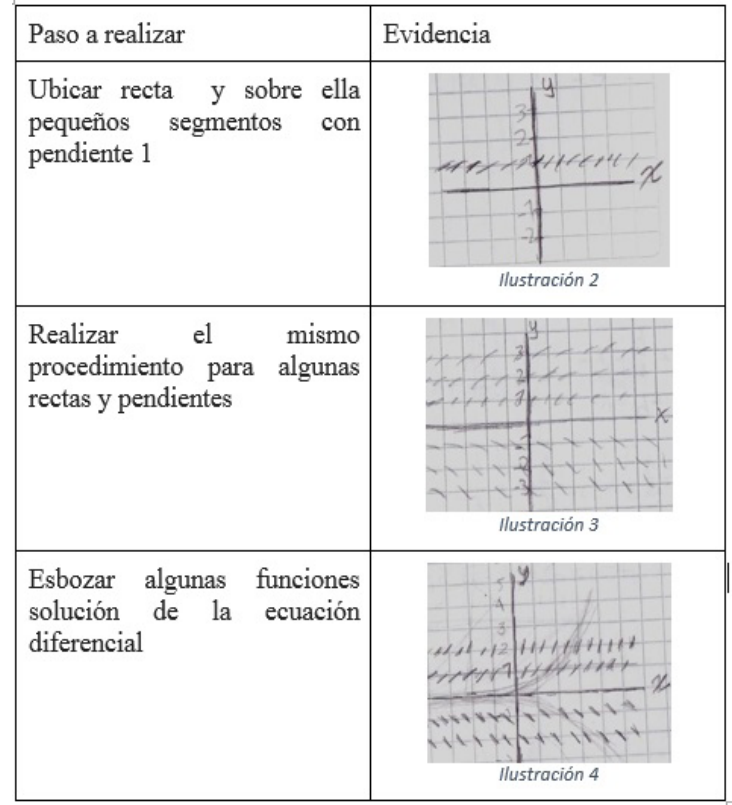

Fuente: Elaboración propia 
Ahora tengamos en cuenta una función que no contenga solamente la variable $y$ sino también $x$, sea la ecuación diferencial $y^{\prime}=1 / x$; en este caso la variable dependiente es $y$ ', es decir que al otorgar valores a $x$ resultarán los de $y^{\prime}$.

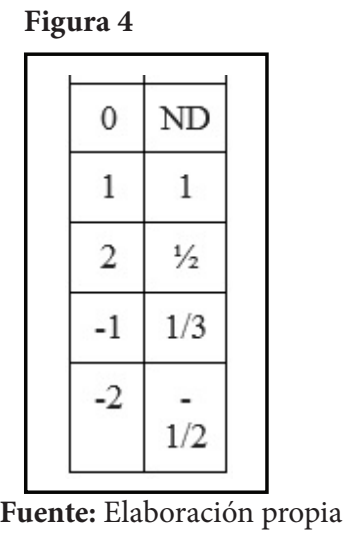

En este caso se puede afirmar que cada $x=n$ es una recta vertical, y se debe hacer el análisis de que por ejemplo, cuando $x=2$, sobre esta recta deben trazarse minúsculos segmentos con pendiente $1 x / 2$ ya que en la tabla cuando $x$ vale 2 su derivada es $1 / 2$.

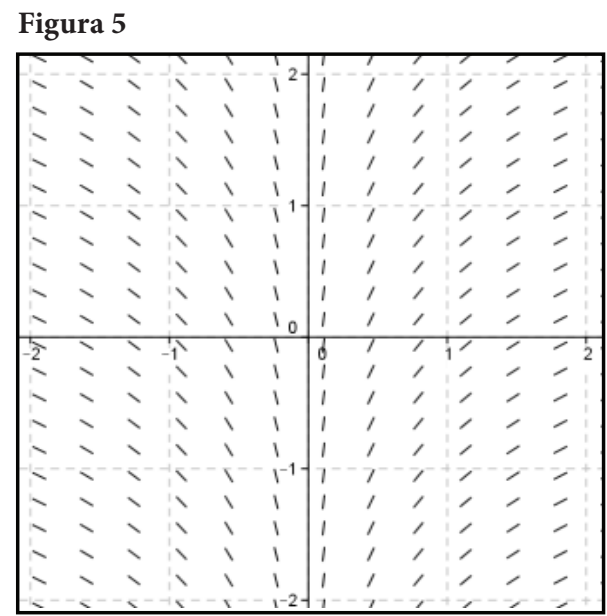

Fuente: Elaboración propia

En este caso también se puede observar que la función no está definida cuando $x$ es cero puesto que la división entre 0 no está definida, es decir que $x=0$ es una asíntota de las soluciones de la ecuación diferencial, realizando los procedimientos del ejemplo anterior se encontrará que el campo direccional es el que se encuentra en la figura 5.

Finalmente se debe tener en cuenta que cualquier ecuación diferencial de primer orden puede ser abordada de manera similar obteniendo los bosquejos de las funciones solución de la ecuación.

\section{Análisis de datos}

Para realizar un análisis de los resultados a la hora de comprobar soluciones de ecuaciones diferenciales sin hacer uso de integrales o derivadas se observará algunos errores que se pueden cometer a la hora de realizar un campo de direcciones:

El primer error puede ser el hecho de no graficar las soluciones teniendo en cuenta quién es la variable dependiente y quién es la independiente.

Otro error puede ser que si la ecuación que se pretende solucionar es por ejemplo $y^{\prime}=x+y$ se tome como si $y$ y $y^{\prime}$ fueran la misma variable, de esta manera no se estará haciendo de manera correcta el campo direccional.

Otro aspecto importante es que en los ejemplos del marco teórico se podía dar solución tabulando y asignando valores cualesquiera en el caso de ecuaciones como $y^{\prime}=x+y$ a la hora de tabular se realizarán análisis similares, pero con objetos diferentes, en este caso la variable independiente es $y^{\prime}$ y de este valor dependen $x=y$ por lo cual si $y=0$ entonces $\mathrm{x}+\mathrm{y}=$ 0 generando la recta, quiere decir que por esta recta se dibujarán diminutos segmentos de pendiente 0 , generando campos direccionales como el siguiente:

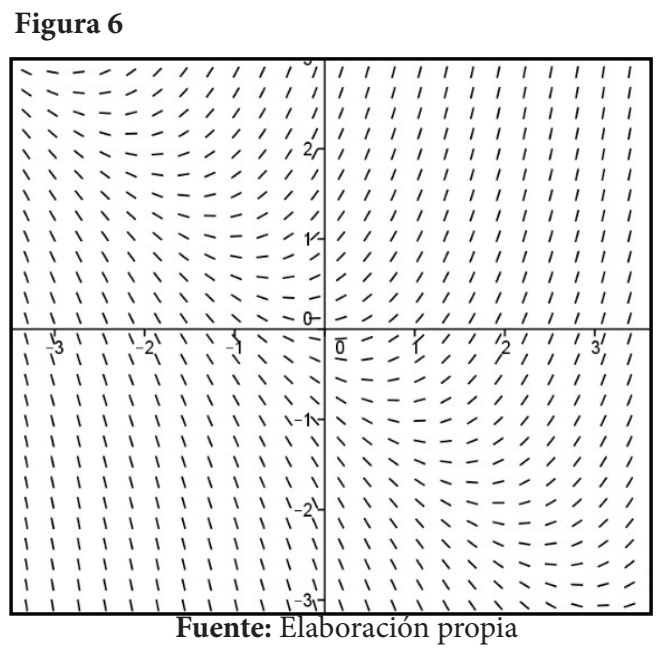


Teniendo en cuenta este campo direccional y el proceso realizado para hacerlo, se puede encontrar la solución de bastantes ecuaciones diferenciales de primer orden.

\section{Conclusiones}

Esta clase de actividades sugiere por parte de los estudiantes para profesor de matemáticas comprender los conceptos que se emplean a la hora de realizar los campos direccionales, logrando establecer relaciones entre los diferentes sistemas de representación, en este caso, entre el gráfico y la tabla.

Analizar las equivocaciones cometidas en las construcciones de los campos de direcciones permite que los estudiantes reconozcan cuáles son los tipos de errores o dificultades que se presentan en este tipo de actividades e identificar cuáles de ellas son inherentes al concepto mismo y cuales son generadas por la errónea identificación de cada ecuación.
Finalmente, el proponer a los estudiantes para profesor este tipo de actividades posibilita reconocer otro tipo de enfoque para la enseñanza de las matemáticas, en el cual se puede hacer uso de diferentes caminos a temáticas complejas como las ecuaciones diferenciales sin pasar por temáticas previas como la derivada y la integral.

\section{Referencias}

Cánova, J. (2004).Teoría cualitativa de ecuaciones diferenciales. En Apuntes de ecuaciones diferenciales. Cartagena: Colombia

Amaya, R. (2006). Un estudio del cambio y la variación a través de su representación gráfica. Colección: Cuadernos de matemática educativa No. 7. Asocolme. 